\title{
Stability of Balanced Implicit Method for Hybrid Stochastic Differential Equation
}

\author{
Sheng $\mathrm{Xu}^{*}$ \\ School of Management, Hefei University of Technology \\ Hefei, China \\ *Corresponding author
}

\author{
Baocang Xia \\ School of Management, Hefei University of Technology \\ Hefei, China
}

\begin{abstract}
The paper shows that the balanced implicit method reproduces the moment exponentially stable for hybrid stochastic differential equation. The balanced implicit method has better behavior of stability in comparison with the explicit Euler-Maruyama method. Experiments simulation indicates that the balanced implicit method may overcome unstability of the Euler-Maruyama method.
\end{abstract}

Keywords-stochastic differential equation, balanced implicit method, markovian switching, moment exponentially stability

\section{INTRODUCTION}

In the 1970 s, Black and Scholes [1] proposed:

$$
\mathrm{d} x(t)=\mu x(t) \mathrm{d} t+\sigma x(t) \mathrm{d} \omega(t)
$$

and he got the Nobel Prize in economics in 1993 for this model. In th is model, the terminology and notation are defined as follows:

\section{$\omega(t)$ :A standard Winner Process, ${ }^{\mu}$ : Asset returns \\ $\sigma$ : Fluctuation ratio}

In the classical Black-Scholes model, $\mu$ and $\sigma$ are constants. While Hull and White [2, 3], Yin and Zhou[4] found $\mu_{\text {and }} \sigma$ are Markov processes instead of constants. So the Markov switching system is proposed:

$$
\mathrm{d} x(t)=\mu(r(t)) x(\mathrm{t}) \mathrm{d} t+\sigma(r(t)) x(t) \mathrm{d} \omega(t),
$$

In this model, $r(t)(t>0)$ is a Markov Chain, and its value ranges is a finite state space $S=\{1,2, \ldots, N\}$.The Markov switching system is popularly used in many financial models, such as in pricing bonds, mortgage bonds, convertible bonds etc.. [5]. However, most of the stochastic differential equation cannot get explicit solution, the numerical method is commonly concerned, various numerical methods are put forward, such as Euler's method, the implicit Euler method, the backward Euler method etc.. [10]. Implicit Euler method with its better stability, gets more attention, especially the

\author{
Shaobo Zhou \\ School of Mathematics and Statistics \\ Huazhong University of Science and Technology \\ Wuhan, China
}

backward Euler method which is often used in the nonlinear systems. But implicit Euler method is only applicable to rigid equation with little noise because this method only partly uses the implicit item in drift coefficient. In this paper, by means of discrete Ito Lemma, we demonstrate that the balanced imp lic it method torque index of markov switching asset pricing model is stable, and at the same time we give the instability condition and conclude that balanced implicit method is more stable than Euler's method with numerical method.

\section{BALANCED IMPLICIT METHOD}

Let $|x|, x \in R^{n}$ be a Euclidean norm, If $A$ is a vector or matrix, its transpose is denoted by $A^{\mathrm{T}}$, and if $A$ is a matrix, its trace norm is denoted by $|A|=\sqrt{\operatorname{trace}\left(A^{\mathrm{T}} A\right)}$, while its operator norm is denoted by $\|A\|=\sup \{|A x|:|x|=1\}$. We let $\left(\Omega, F,\left(F_{t}\right)_{t \geq 0}, P\right)$ be a complete probability space with a filtration $\left(F_{t}\right)_{t \geq 0}$,satisfying the usual conditions (i.e. incremental, right of continuous, $\boldsymbol{F}_{\mathrm{o}}$ Contains all the zero measure set); $\omega(t)$ is a standard Brown motion in probability space; $r(t)(t \geq 0)$ is a right continuous markov chain whose value range is the finite state space $S=\{1,2, \ldots, N\}$,it generates $\Gamma=\left(\gamma_{\mathrm{ij}}\right)_{N \times N}$ and satisfies

$$
P\{r(t+\Delta)=j \mid r(t)=i\}= \begin{cases}\gamma_{i j} \Delta+0(\Delta), & \text { if } i \neq j, \\ 1+\gamma_{i i} \Delta+0(\Delta), & \text { if } i=j,\end{cases}
$$

where $\Delta>0, \gamma_{i j} \geq 0$ is the transition probability from $i$

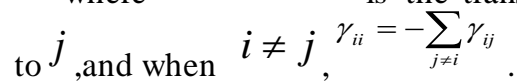

Considering that $r(t)$ is a right successive step function with limited multiple jump discontinuity point in $\boldsymbol{R}_{+}$, we assume that the markov chain $r(t)$ is independent of Brownian movement $\omega(t)$. We also assume that the markov chain is irreducible, that is for any $i, j \in S$, exists 
$i_{1}, i_{2}, \cdots, i_{k} \in S$, which can make sure $\gamma_{i, i_{1}} \gamma_{i_{1}, i_{2}} \cdots \gamma_{i_{k}, j}>0$ and 0 is an Eigen value root of $\Gamma$, and ${ }^{\operatorname{rank}}(\Gamma)=N-1$.So there is a unique stationary distribution $\pi=\left(\pi_{1}, \pi_{2}, \cdots, \pi_{N}\right) \in R^{1 \times N}$, which satisfies $\pi \Gamma=0, \sum_{j=1}^{N} \pi_{j}=1, \pi_{j}>0, j \in S$.

Lemma 2.1([6]) We assume that for any $\Delta>0, k \geq 0, r_{k}^{\Delta}=r(k \Delta)$, then $\left\{r_{k}^{\Delta}, k=0,1,2 \ldots\right\}$ is a discrete markov chain, whose One-step transition probability matrix array is $P(\Delta)=\left(P_{i j}(\Delta)\right)_{N \times N}=e^{\Delta \Gamma}$.

Given the Linear markov switching stochastic differential equation:

$$
\mathrm{d} x(t)=\mu(r(t)) x(t) \mathrm{d} t+\sigma(r(t)) x(t) \mathrm{d} \omega(t),
$$

on $\quad t \geq 0, x(0)=x_{0} \in R, r(0)=r_{0} \in S, \quad$ and $\quad r_{0}$ is a measurable random variables of $S$-Value $F_{t}$.

Since $\gamma_{i j}$ is independent of $x$, and the path of $r$ is produced before $x$ and independent of $x$. We define $\Delta \in(0,1)$ $\left\{r_{k}^{\Delta}, k=0,1,2, \ldots\right\}$ as follows: calculate the one-step transition probability matrix $P(\Delta)$.Let $r_{0}^{\Delta}=i_{0}$ and get a random number $\xi_{1}$ which distributed evenly over [0,1].Then we define

$$
r_{1}^{\Delta}=\left\{\begin{array}{l}
i_{1} \quad i_{1} \in S-\{N\}, \sum_{j=1}^{i_{1}-1} P_{i, j}(\Delta) \leq \xi_{1}<\sum_{j=1}^{i_{1}} P_{i_{0}, j}(\Delta), \\
N, \quad \sum_{j=1}^{N-1} P_{i_{i, j}}(\Delta) \leq \xi_{1},
\end{array}\right.
$$

on $\sum_{j=1^{i, j}}^{O}(\Delta)=0$. which is distributed evenly over $[0,1]$ and is independent of $\xi_{1}$. We define

$$
r_{2}^{\Delta}=\left\{\begin{array}{c}
i_{2} \quad i_{2} \in S-\{N\}, \sum_{j=1}^{i_{2}-1} P_{r_{r}, j}(\Delta) \leq \xi_{2}<\sum_{j=1}^{i_{2}} P_{r_{r}, j}(\Delta), \\
N, \quad \sum_{j=1}^{N-1} P_{P_{r, j}, j}(\Delta) \leq \xi_{2} .
\end{array}\right.
$$

Repeating this process, we can get $\left\{r_{k}^{\Delta}, k=1,2, \ldots\right\}$.This process can independently produce more trajectory, after we get the discrete markov chain $\left\{r_{k}^{\Delta}, k=1,2, \ldots\right\}$, we define the balanced implicit method of hybrid system (3).For $t_{k}=k \Delta, k>0 . X_{0}=x\left(t_{0}\right), r_{0}^{\Delta}=i_{0}$, here is the approximate value for $X_{k} \approx x\left(t_{k}\right)$ :

$$
X_{k+1}=X_{k}+\mu\left(r_{k}^{\Delta}\right) X_{k} \Delta+\sigma\left(r_{k}^{\Delta}\right) X_{k} \Delta \omega_{k}+C\left(X_{k}\right)\left(X_{k}-X_{k+1}\right),
$$

where $\Delta \omega_{k}=\omega\left(t_{k+1}\right)-\omega\left(t_{k}\right)_{\text {is a }} F_{t_{k}}$-measurable random variable which is independent and Obey the $N(0, \Delta)$ distribution. $C\left(X_{k}\right)$ satisfies $\quad C\left(X_{k}\right)=c_{0 k} \Delta+c_{1 k}\left|\Delta \omega_{k}\right|$, $c_{0 k}=C_{0}\left(X_{k}\right), \quad c_{1 k}=C_{1}\left(X_{k}\right)$ is known as control function, and often is chosen as constant[7].

Hypothesis 2.2: Assume that $c_{0 k}$ and $c_{1 k}$ are limited function, for any step size $\Delta, \bar{\alpha}_{0} \geq \Delta, \alpha_{0} \in\left[0, \bar{\alpha}_{0}\right]$, $\alpha_{1} \geq 0, \quad M(x)=1+\alpha_{0} c_{0 k}(x)+\alpha_{1} c_{1 k}(x)$

$\left|(M(x))^{-1}\right| \leq C<\infty, C>0$. The equilibrium methods (4)is very general, it covers the implicit and semi implicit Euler method. If denoted by $C\left(X_{k}\right)=(\theta-1) \mu\left(r_{k}^{\Delta}\right) \Delta, \quad$ and from (4), we can get the following semi implicit method

$$
X_{k+1}=X_{k}+\theta \mu\left(r_{k}^{\Delta}\right) X_{k} \Delta+(1-\theta) \mu\left(r_{k}^{\Delta}\right) X_{k+1} \Delta+\sigma\left(r_{k}^{\Delta}\right) X_{k} \Delta \omega_{k},
$$

then for $\theta=1$, we can get the Euler's method

$$
X_{k+1}=X_{k}+\mu\left(r_{k}^{\Delta}\right) X_{k} \Delta+\sigma\left(r_{k}^{\Delta}\right) X_{k} \Delta \omega_{k},
$$

Next we assume that $c_{0 k}, c_{1 k}$ is independent of the constant $k$,and $c_{0}=c_{0 k}, \quad c_{1}=c_{1 k}$.

It is easy to prove that balanced implicit method and Euler method have strong convergence with the same $1 / 2$ order. Th is article will take all the constants and all the product with constants as $C$ except $c_{0}, c_{1}$,

\section{ST ABILITY}

Lemma 3.1([6]) we assume that ${ }^{\mu}$ is a random variables of standard normal distribution, $\phi(x) \in C^{3}(R),\left|\phi^{\prime \prime \prime}(x)\right| \leq L$, and $L \quad$ is a constant $x \in[1-\delta, 1+\delta](0<\delta<1), \phi: R \rightarrow R$ is a Lebesgue integrable function, then for $c_{1}, c_{2}, c_{3} \in R, \Delta \rightarrow 0$,

$$
\begin{aligned}
& E\left[\phi\left(1+c_{1} \Delta+c_{2} \sqrt{\Delta} \mu+c_{3} \sqrt{\Delta}|\mu|\right)\right]=\phi(1)+ \\
& \phi(1)\left(c_{1} \Delta+\frac{2}{\sqrt{2 \pi}} c_{3} \sqrt{\Delta}\right)+\frac{\phi(1)}{2}\left(c_{2}^{2}+c_{3}^{2}\right) \Delta+o(\Delta) .
\end{aligned}
$$


Theorem 3.2 Assume that there is a positive constant $\Delta^{*}$, for any $\Delta<\Delta^{*}$, from the balanced implicit method (4) we can get

$\lim \sup _{n \rightarrow \infty} \frac{1}{n \Delta} \log E\left|X_{n}\right|^{p} \leq \frac{p}{\Delta} \log C+\sum_{j \in S} \pi_{j} p\left[\begin{array}{l}\frac{4}{\sqrt{2 \pi}} c_{1} \Delta^{-\frac{1}{2}}+\mu_{j}+c_{0} \\ +\frac{p-1}{2}\left(\sigma_{j}^{2}+c_{1}^{2}\right)+C \Delta^{\frac{1}{2}}+o\left(\Delta^{\frac{1}{2}}\right)\end{array}\right]$.

that is, for a sufficiently small step size $\Delta$,if

$$
\frac{p}{\Delta} \log C+\sum_{j \in S} \pi_{j} p\left[\begin{array}{l}
\frac{4}{\sqrt{2 \pi}} c_{1} \Delta^{-\frac{1}{2}}+\mu_{j}+c_{0} \\
+\frac{p-1}{2}\left(\sigma_{j}^{2}+c_{1}^{2}\right)+C \Delta^{\frac{1}{2}}+o\left(\Delta^{\frac{1}{2}}\right)
\end{array}\right]<0
$$

then the balanced implicit method(4)is $p$-th moment exponential stable.

Proof From the sequence(4), we can get

$$
E\left[\left|X_{n+1}\right|^{p}\right]=E\left(\left|X_{0}\right|^{p} \prod_{k=0}^{n-1} Z_{k}^{p} E\left[Z_{n}^{p} \mid F_{t_{n}}\right]\right),
$$

$$
F_{t_{n}}=\sigma\left(\{r(u)\}_{u \geq 0,}\{\omega(s)\}_{0 \leq s \leq t_{n}}\right) \text { is a } \sigma_{\text {-algebra, produced }}
$$

by $\{r(u)\}_{u \geq 0 \text {, and }}\{\omega(s)\}_{0 \leq s \leq t_{n}}$,through the assumption, calculate that

$$
\begin{aligned}
Z_{n}^{p} & =\left|\frac{1+c_{0} \Delta+c_{1}\left|\Delta \omega_{n}\right|+\mu\left(r_{n}^{\Delta}\right) \Delta+\sigma\left(r_{n}^{\Delta}\right) \Delta \omega_{n}}{1+c_{0} \Delta+c_{1}\left|\Delta \omega_{n}\right|}\right|^{p} \\
& \leq C^{p}\left\{\begin{array}{l}
\left.1+2\left[\left(c_{0}+\mu\left(r_{n}^{\Delta}\right)\right) \Delta+c_{1}\left|\Delta \omega_{n}\right|+\sigma\left(r_{n}^{\Delta}\right) \Delta \omega_{n}\right]\right\}^{\frac{p}{2}} \\
+\left[\left(c_{0}+\mu\left(\gamma_{n}^{\Delta}\right)\right) \Delta+c_{1}\left|\Delta \omega_{n}\right|+\sigma\left(r_{n}^{\Delta}\right) \Delta \omega_{n}\right]
\end{array}\right] .
\end{aligned}
$$

and from the Taylor expansion, let

$$
\begin{gathered}
u=2\left[\left(c_{0}+\mu\left(r_{n}^{\Delta}\right)\right) \Delta+c_{1}\left|\Delta \omega_{n}\right|+\sigma\left(r_{k}^{\Delta}\right) \Delta \omega_{n}\right] \\
+\left[\left(c_{0}+\mu\left(r_{n}^{\Delta}\right)\right) \Delta+c_{1}\left|\Delta \omega_{k}\right|+\sigma\left(r_{n}^{\Delta}\right) \Delta \omega_{n}\right]^{2}
\end{gathered}
$$

and choose $\Delta, c_{0}, c_{1}$, which can make sure that $u>-1$, then

$$
E\left[Z_{n}^{p} \mid F_{t_{n}}\right] \leq C^{p} E\left[\begin{array}{l}
1+\frac{p}{2} u+\frac{p(p-2)}{8} u^{2} \\
+\frac{p(p-2)(p-4)}{48} u^{3} \mid F_{t_{n}}
\end{array}\right] .
$$

Let $u_{1}=\left(c_{0}+\mu\left(r_{n}^{\Delta}\right)\right) \Delta+c_{1}\left|\Delta \omega_{n}\right|+\sigma\left(r_{k}^{\Delta}\right) \Delta \omega_{n}$, then $u=2 u_{1}+u_{1}^{2} \cdot$ It is easy to calculate that

$$
\begin{aligned}
& E\left[\left(\Delta \omega_{n}\right)^{2 i}\right]=(2 i-1) ! ! \Delta^{i}, E\left[\left(\Delta \omega_{n}\right)^{2 i-1}\right]=0, \\
& E\left[\left|\Delta \omega_{n}\right|^{2 i-1}\right]=\frac{2^{i}}{\sqrt{2 \pi}}(i-1) ! \Delta^{\frac{2 i-1}{2}},
\end{aligned}
$$

$$
\begin{gathered}
\text { on } \begin{array}{l}
(2 i-1) ! !=(2 i-1)(2 i-3) \cdots 3 \cdot 1, \\
(i-1) !=(i-1)(i-2) \cdots 2 \cdot 1, i=1,2 \cdots .
\end{array} \\
E\left[\mu_{1} \mid F_{t_{n}}\right]=\frac{2}{\sqrt{2 \pi}} c_{1} \Delta^{\frac{1}{2}}+\left(c_{0}+\mu\left(r_{n}^{\Delta}\right)\right) \Delta,
\end{gathered}
$$

$$
\begin{aligned}
& E\left[\mu_{1}^{2} \mid F_{t}\right]=\left(c_{1}^{2}+\sigma^{2}\left(r_{n}^{\Delta}\right)\right) \Delta+\frac{4}{\sqrt{2 \pi}} c_{1}\left(c_{0}+\mu\left(r_{n}^{\Delta}\right)\right) \Delta^{\frac{3}{2}} \\
& +\left(c_{0}+\mu\left(r_{n}^{\Delta}\right)\right)^{2} \Delta^{2},
\end{aligned}
$$

$$
E\left[\mu_{1}^{3} \mid F_{t}\right]=C \Delta^{\frac{3}{2}}+o\left(\Delta^{\frac{3}{2}}\right), E\left[\mu_{1}^{4} \mid F_{t_{t}}\right]=o\left(\Delta^{\frac{3}{2}}\right) .
$$

plug (10)-(12) into (5), then

$$
E\left[\left|X_{n+1}\right|^{p}\right] \leq E\left(\left|X_{0}\right|^{p} C^{p}\left(\begin{array}{c}
1+\frac{2}{\sqrt{2 \pi}} p c_{1} \Delta^{\frac{1}{2}} \\
\left.+p\left(\begin{array}{l}
\mu\left(r_{n}^{\Delta}\right)+c_{0} \\
+\frac{p-1}{2}\left(\begin{array}{l}
\sigma^{2}\left(r_{n}^{\Delta}\right) \\
+c_{1}^{2}
\end{array}\right)
\end{array}\right] \Delta\right) \prod_{k=0}^{n-2} Z_{k} E\left[Z_{n-1} \mid F_{t-1}\right] \\
+C \Delta^{\frac{3}{2}}+o\left(\Delta^{\frac{3}{2}}\right)
\end{array}\right)\right.
$$

Repeat this process, then

$$
\begin{aligned}
& E\left[\left|X_{n+1}\right|^{p}\right] \leq E\left(\left|X_{0}\right|^{p} \exp \left[+\sum_{k=0}^{n} \log \left(\begin{array}{l}
1+\frac{2}{\sqrt{2 \pi}} p c_{1} \Delta^{\frac{1}{2}} \\
\left.\left[\begin{array}{l}
\mu\left(r_{k}^{\Delta}\right)+c_{0} \\
+\frac{p-1}{2}\left(\sigma^{2}\left(r_{k}^{\Delta}\right)+c_{1}^{2}\right)
\end{array}\right] \Delta\right)+o\left(\frac{\log C}{\left.\Delta^{\frac{3}{2}}\right)}\right. \\
+C \Delta^{\frac{3}{2}}
\end{array}\right]\right)\right. \\
& \text { By markov chain ergodicity and the inequation } \log (1+x) \leq x, \\
& \forall x>-1 \text { we can figure out }
\end{aligned}
$$




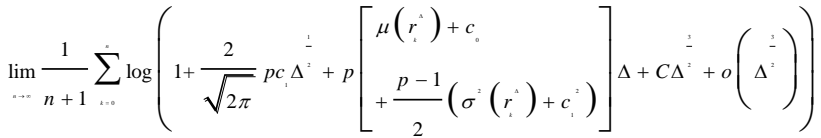

$$
\begin{aligned}
& \leq \sum_{i=s} \pi p\left[\frac{2}{\sqrt{2 \pi}} c \Delta^{2}+\left[\mu+\frac{p-1}{2} \sigma^{2}\right] \Delta+\left(c_{0}+\frac{p-1}{2} c^{2}\right) \Delta+C \Delta^{2}+o\left(\Delta^{\prime}\right)\right] \text { a.s. }
\end{aligned}
$$

Denoted by

$$
\lambda=\frac{p}{\Delta} \log C+\sum_{j \in S} \pi_{j} p\left[\begin{array}{l}
\frac{4}{\sqrt{2 \pi}} c_{1} \Delta^{-\frac{1}{2}}+\mu_{j}+c_{0} \\
+\frac{p-1}{2}\left(\sigma_{j}^{2}+c_{1}^{2}\right)+C \Delta^{\frac{1}{2}}+o\left(\Delta^{\frac{1}{2}}\right)
\end{array}\right] .
$$

then there exists $\varepsilon>0$, which make

$$
\lim _{n \rightarrow \infty} \frac{1}{n+1}\left[+\sum_{k=0}^{n} \log \left(\begin{array}{l}
p(\mathrm{n}+1) \log C \\
\left.1+\frac{2}{\sqrt{2 \pi}} p c_{1} \Delta^{\frac{1}{2}}+p\left[\begin{array}{l}
\mu\left(r_{k}^{\Delta}\right)+c_{0} \\
+\frac{p-1}{2}\left(\sigma^{2}\left(r_{k}^{\Delta}\right)+c_{1}^{2}\right)
\end{array}\right] \Delta\right] \\
+C \Delta^{\frac{3}{2}}+o\left(\Delta^{\frac{3}{2}}\right)
\end{array}\right]\right.
$$

$<\lambda+\varepsilon$ a.s.

According to the lemma Fatou,$$
\text { degenerates to } \sum_{j \in S} \pi_{j} p\left(\mu_{j}+\frac{p-1}{2} \sigma_{j}^{2}\right)<0
$$

$$
\lim _{n \rightarrow \infty} e^{-(\lambda+\varepsilon)(n+1) \Delta} E\left|X_{n+1}\right|^{p}=0
$$

If choose

$$
c_{1}<0, c_{0}+\frac{p-1}{2} c_{1}^{2}<0, C<1,
$$

then criteria $(5)$

This is the sufficient condition for $p_{\text {-th }}$ moment exponential stability of Eu ler's method.

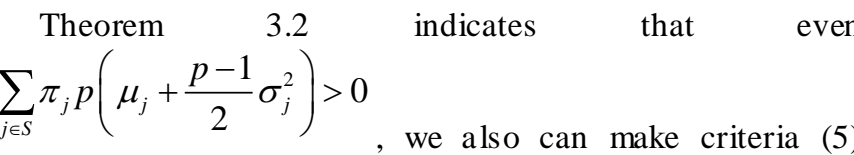

possible when the controls parameters $c_{1}, c_{0}$ and $\Delta$ are chosen properly. Combined with the Eu ler's method., balanced implicit method is more stable.

Next is the sufficient condition for instability of method

Theorem 3.3 Balanced implicit method (4)satisfies

$\lim _{n \rightarrow \infty} \frac{1}{n \Delta} \log \left(E\left|X_{n}\right|^{p}\right) \geq \sum_{j \in S} p \pi_{j}\left(\mu_{j}-\frac{1}{2} \sigma_{j}^{2}\right)$. That is if

$\sum_{j \in S} \pi_{j}\left(\mu_{j}-\frac{1}{2} \sigma_{j}^{2}\right)>0$, for any sufficiently s mall step size $\Delta$,

balanced implicit method is torque index is not stable.

Proof from (4), we can get

$$
\begin{gathered}
\left|X_{n+1}\right|^{p}=\left|X_{0}\right|^{p} \prod_{k=0}^{n} Z_{k}^{p}=\left|X_{0}\right|^{p} \exp \left(\sum_{k=1}^{n} \log Z_{k}^{p}\right) . \\
E\left[\left|X_{n+1}\right|^{p}\right]=E\left(\begin{array}{l}
\left|X_{0}\right|^{p} \exp \left(\sum_{k=1}^{n-1} \log Z_{k}^{p}\right) \\
E\left[\exp \left(\log Z_{k}^{p}\right) \mid F_{t_{n}}\right]
\end{array}\right) .
\end{gathered}
$$

Depend on the Jensen inequation, we can get

$$
E\left[\exp \left(\log Z_{n}^{p}\right) \mid F_{t_{n}}\right] \geq \exp \left(E\left[\log Z_{n}^{p} \mid F_{t_{n}}\right]\right)
$$

use the lemma 3.1, and combine (23), we can get

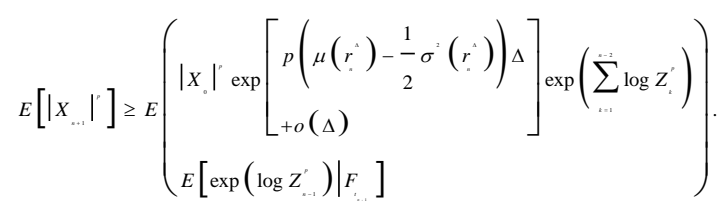

Repeat this process, we find

$$
E\left[\left|X_{n+1}\right|^{p}\right] \geq E\left[\left|X_{0}\right|^{p} \exp \left(\sum_{k=0}^{n}\left[p\left(\begin{array}{c}
\mu\left(r_{k}^{\Delta}\right) \\
-\frac{1}{2} \sigma^{2}\left(r_{k}^{\Delta}\right)
\end{array}\right) \Delta+o(\Delta)\right]\right] .\right.
$$

Given (17), when $\varepsilon>0$, we can get

$$
e^{-(\lambda-\varepsilon) n \Delta} E\left|X_{n}\right|^{p} \geq E\left|X_{0}\right|^{p} \exp \left[\begin{array}{l}
-(\lambda-\varepsilon) n \Delta \\
+\sum_{k=0}^{n} p\left(\mu\left(r_{k}^{\Delta}\right)-\frac{1}{2} \sigma^{2}\left(r_{k}^{\Delta}\right)\right) \Delta+o(\Delta)
\end{array}\right] .
$$

Depend on the ergodicity of Markov chain, we can get

$$
\begin{aligned}
& \lim _{n \rightarrow \infty} \frac{1}{n \Delta} \sum_{k=1}^{n}\left[p\left(\mu\left(r_{k}^{\Delta}\right)-\frac{1}{2} \sigma^{2}\left(r_{k}^{\Delta}\right)\right) \Delta+o(\Delta)\right]=\sum_{j \in S} \pi_{j}\left[p\left(\mu_{j}-\frac{1}{2} \sigma_{j}^{2}\right)+o(\Delta)\right] . \\
& \text { Let } \lambda=\sum_{j \in S} \pi_{j} p\left(\mu_{j}-\frac{1}{2} \sigma_{j}^{2}\right), \\
& \text { then }
\end{aligned}
$$

So $\lim \inf _{n \rightarrow \infty} \frac{1}{n \Delta} \log E\left|X_{n}\right|^{p} \geq \sum_{j \in S} \pi_{j} p\left(\mu_{j}-\frac{1}{2} \sigma_{j}^{2}\right)-\varepsilon$.

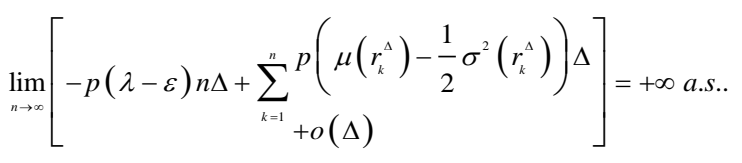

when $\varepsilon \rightarrow 0$, then

$$
\lim \inf _{n \rightarrow \infty} \frac{1}{n \Delta} \log E\left|X_{n}\right|^{p} \geq \sum_{j \in S} \pi_{j} p\left(\mu_{j}-\frac{1}{2} \sigma_{j}^{2}\right) .
$$


Theorem 3.3 indicates that the condition of instability for method (4) is stronger than Euler's, that's balanced implic it method has better stability than Euler's method.

\section{NUMERICAL EXPERIMENT}
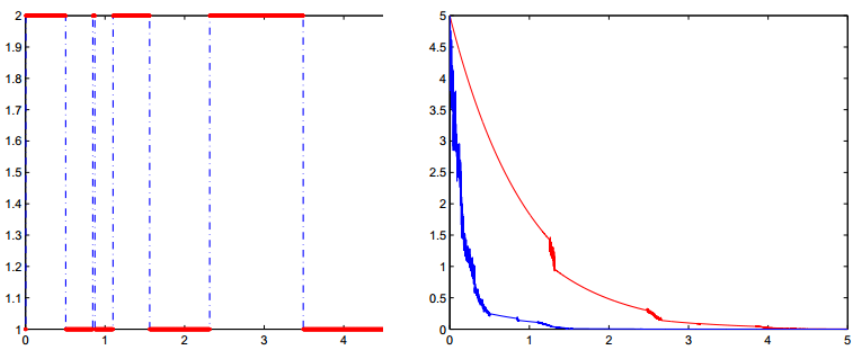

FIGURE I. LEFT:THE PATH OF $r(t)$; RIGHT:THE PATH OF EULER'S METHOD(RED LINE)AND BALANCED IMPLICIT MET HOD (BLUE LINE) WHEN THE STEP SIZE $\Delta=0.001$

In this section we will verify the correctness of the conclusion, through the Numerical experiment we confirmed that the balanced imp lic it method has better stability. Consider a scalar equation

$$
\mathrm{d} x(\mathrm{t})=\mu(r(\mathrm{t})) x(\mathrm{t}) \mathrm{d} t+\sigma(r(\mathrm{t})) x(\mathrm{t}) \mathrm{d} \omega(\mathrm{t}),
$$

and in it $t \geq 0, \omega(t)$ is scalar Brownian motion, $r(t)$ is a right successive markov chain which values in $S=\{1,2\}$,and generates $\Gamma=\left(\begin{array}{cc}-1 & 1 \\ 2 & -2\end{array}\right)$.

Assume that $r(t)_{\text {is independent of }} \omega(t)$.It is easy to know $\pi_{1}=\frac{2}{3}, \pi_{2}=\frac{1}{3}$. and when $(x(0), r(0))=(5,1)$, the horizontal axis represents time variable ${ }^{t}$.In the figure 1,2 and 4, Red line represents the curve orbit of Euler's method, the blue line represents the orbit curve of balanced implicit method. In figure 3 , red line represents the curve orbit of balanced implicit method when $c_{0}=-1, c_{1}=-2$ while the blue line represents the orbit curve of balanced implicit method when $c_{0}=-0.1, \quad c_{1}=-0.2$.
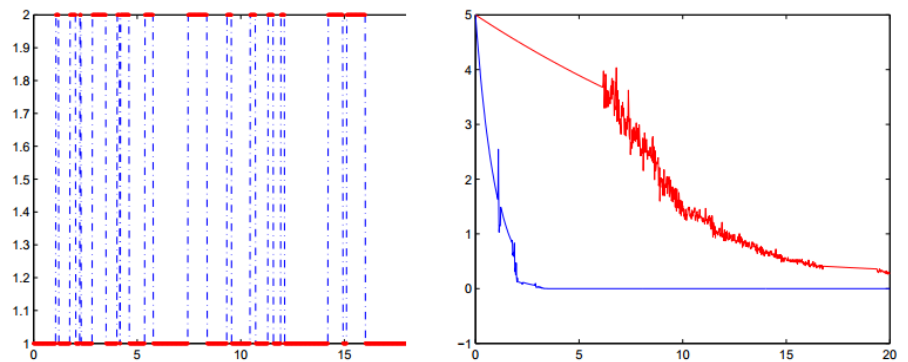

FIGURE II. LEFT: THE PAT H OF ${ }^{r(t)}$ : RIGHT; THE PAT H OF EULER'S METHOD(RED LINE)AND BALANCED IMPLICIT MET HOD (BLUE LINE) WHEN THE STEP SIZE $\Delta=0.001$
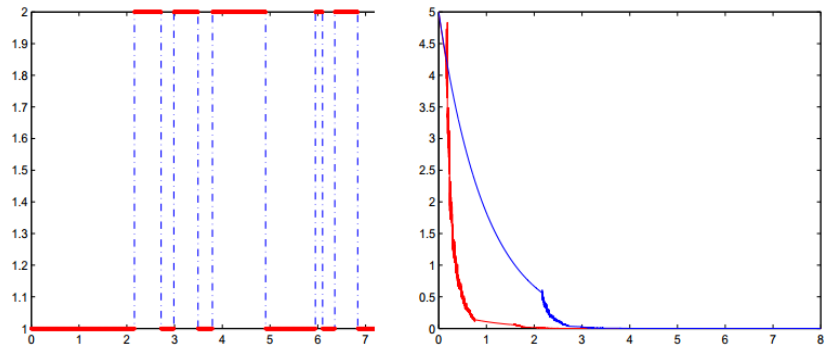

FIGURE III.LEFT:THE PATH OF $r(t)$; RIGHT:WHEN THE STEP SIZE IS $\Delta=0.001, T=8$. THE RED LINE REPRESENT STHE PATH OF BALANCED IMPLICT METHOD WHEN $c_{\mathrm{O} n}=-1, c_{1 n}=-2$,AND BLUE LINE REPRESENT S THEPATH OF BALANCED IMPLICIT

$$
\text { METHODWHEN } c_{0 n}=-0.1, c_{1 n}=-0.2
$$

Case $1 \mu(1)=-1, \sigma(1)=0, \sigma(2)=2$

In this condition, we can find $\sum_{j \in S} \pi_{j} p\left(\mu_{j}-\frac{1}{2} \sigma_{j}^{2}\right)=-\frac{4}{3}$, which indicate that the equation(27)is almost asymptotic stable everywhere, figure 1 include the Orbit curve for both Euler's method(when $\Delta=0.001, T=5, \quad c_{0}=0, \quad c_{1}=0$ ) and the balanced implicit method (when $\Delta=0.001 T=5$, $c_{0}=-0.1, \quad c_{1}=-2$ ); figure 2 include the Orbit curves for both Euler's method and the balanced implicit method when $h=0.001, T=20, \quad c_{0 n}=-0.1, c_{1}=-0.2$.These two figures indicate that balanced implicit method has better stability than Euler's method. In figure 3, we can find the Orbit curves for balanced implicit method when $h=0.001, T=8$ and control coefficient respectively are $c_{0}=-1, c_{1}=-2$ and $c_{0}=-0.1, \quad c_{1}=-0.2$, which indicate that the stability can be influenced by the control coefficients $c_{0}$ and $c_{1}$.

$$
\text { Case } 2 \mu(1)=1.2, \sigma(1)=1, \mu(2)=1.2, \sigma(2)=1 \text {. }
$$
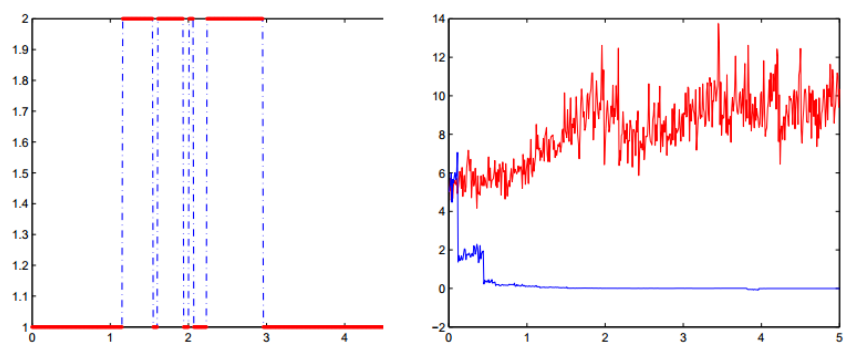

FIGURE IV. LEFT:THE PAT H OF ${ }^{r(t)}$; RIGHT:THE CURVE OF EULER'S METHOD(RED LINE)AND BALANCED IMPLICTT METHOD (BLUE LINE) WHEN $h=0.001, T=5, c_{0}=-1, c_{1}=-2$ 
We can figure out that $\sum_{j \in S} \pi_{j} p\left(\mu_{j}-\frac{1}{2} \sigma_{j}^{2}\right)>0$, and Euler's method is not stable. Figure 4 shows orbital curve of balanced implicit method and Euler's method when $h=0.001, T=5, c_{0}=-1, \quad c_{1}=-2$,we can draw a conclusion from the figure that balanced implicit method is stable, while Euler's method is not, that is balanced implicit method can keep stable by choosing appropriate control coefficient, this further illustrates that the balanced implicit method can overcome the instability of Euler method, and makes the system stable.

\section{REFERENCES}

[1] F.Black, M. Scholes, The price of options and corporate liabilities, J. of Political Economy, 1973 vol 81:)637-654.

[2] J. Hull, White A. The pricing of options on assets with stochastic volatilities, Journal of Fiance,1987 vol 42: 281-300.
[3] Hull,J., and A.White, Pricing interest rate derivative securities, Review of Financial Studies, 1990 vol 3: 573-592.

[4] G.G. Yin, X.Y. Zhou, Markovian mean-variance port folio selection with regime switching: from discrete-time models to theircontinuous-time limits, IEEE Transactions on Automatics Control,2004 vol 49,349-360.

[5] Zhongfei ,Jinyao Gao, General equilibrium pricing under the condition of model uncertainty, System engineering theory practice,2011 Vol. 31 (12): 2272-2280.

[6] L. Hu, S Gan, X Wang, Asymptotical stability of the balanced methods for stochastic jump-diffusion differential equations, Journal of Computational and Applied Mathematics, in press.

[7] G. N. Milstein, P.E. Schurz, Balanced implicit methods for stiff stochastic systems, SIAM J Numer.Anal. 1998 vol 35: 1010-1019.

[8] Genser. D.M. A structural framework for the pricing of corporate securities, Springer-Verlag Berlin Heidelberg 2006.

[9] M. Athans, Command and control(C2)theory: A challenge to control science, IEEE Transactions on Automatic Control 1998 vol32: 286-293.

[10] D.J. Higham, Mean-square and asymptotic stability of stochastic theta method, SIAM J.Numer. Anal. 2000 vol 38: 753-769. 